\title{
Einleitung in das Themaheft «Beiträge der Wirtschafts- und Humangeographie zur Lösung von Umweltproblemen»
}

Der 48. Deutsche Geographentag, der vom 23. bis 26. September 1991 in Basel stattfindet, ist dem Generalthema «Geographie und Umwelt» gewidmet. Die beiden deutschsprachigen schweizerischen Geographie-Zeitschriften, Regio Basiliensis und Geographica Helvetica, haben sich entschlossen, dieser Großveranstaltung der (deutschsprachigen) Geographie je ein Themaheft zu widmen. In Absprache mit weiteren geographischen Publikationsorganen, Geographische Rundschau und Berichte zur deutschen Landeskunde, wurden auch die inhaltlichen und autorenmäßigen Schwerpunkte koordiniert.

Die Geographica Helvetica setzt sich zum Ziel, am Beispiel von theoretischen und empirischen Untersuchungen aufzuzeigen, daß bei der Lösung aktueller Umweltprobleme nicht nur von der Physischen Geographie, sondern auch von der Wirtschafts- und Humangeographie Beiträge zu erwarten sind. Die Aussage, da $\beta$ bei der Behandlung von Umweltproblemen in Forschung, Unterricht und Praxis die traditionellen Disziplingrenzen zwischen Naturwissenschaften einerseits und Sozial- und Wirtschaftswissenschaften anderseits, aber auch diejenigen zu den Ingenieurwissenschaften und Rechtswissenschaften im Sinne einer transdisziplinären Zusammenarbeit überwunden und aufgelöst werden müssen, wird zwar immer wieder bestätigt, die Realität vermittelt allerdings (leider) ein anderes Bild. Die vor mehr als zwanzig Jahren von BARTELS vor dem damaligen Hintergrund der Situation der (deutschen) Geographie zu Recht geforderte und später auch von anderen Autoren, beispielsweise BAHRENBERG, mit Vehemenz vertretene Zweiteilung der Disziplin in eine physischgeographische Ökosystemforschung und in eine sozialwissenschaftliche Regionalforschung muß heutzutage angesichts der drängenden Umweltprobleme kritisch hinterfragt werden. Heute interessieren nicht allein geographische Ansätze in der Umweltforschung einerseits und der Regionalforschung anderseits, sondern es geht auch um den
Beitrag der Geographie im Grenzbereich zwischen Regional- und Umweltforschung. Der Versuch einer Gliederung dieses Grenzbereiches, die es erlaubt, entsprechende geographische Arbeiten einzuordnen, findet sich bei WACHTER. Der Geograph und die Geographin müssen den Mut haben, in diesem anspruchsvollen Grenzbereich aktiv zu werden. Geschieht dies nicht, so müßte einmal mehr von einer verpaßten Chance unserer Disziplin gesprochen werden. Gerade hier trifft die Aussage von BAHRENBERG zu: «Geographie wird sein, was wir tun werden.»

Bei der Autorin und den Autoren des vorliegenden Heftes handelt es sich mehrheitlich um jüngere Geographinnen und Geographen, die meist an geographischen Hochschulinstituten in der deutschsprachigen Schweiz wissenschaftlich tätig sind.

Diese Auswahl darf nun aber keinesfalls den Eindruck erwecken, daß nur in der Deutschschweiz sich die Human- und Wirtschaftsgeographie mit Umweltproblemen beschäftigt. Im Gegenteil, gerade an den Westschweizer Universitäten, wo die Geographie traditionellerweise nicht zu den Naturwissenschaften, sondern zu den Geistes- und Sozialwissenschaften gezählt wird, finden äußerst anregende und zukunftsweisende Diskussionen innerhalb und außerhalb der Geographie bezüglich der Interdependenzen zwischen Umwelt- und Regionalforschung statt. Da das vorliegende $\mathrm{Heft}$ der Geographica Helvetica dem Deutschen Geographentag in Basel gewidmet ist, wurden aber bewußt nur Autoren aus der Deutschschweiz und somit deutschsprachige Artikel berücksichtigt. Die Geographinnen und Geographen aus der Westschweiz mögen dies dem Organisator des Heftes verzeihen; sie dürfen versichert sein, daß die getroffene Auswahl nichts mit deutschschweizerischer oder gar zürcherischer Überheblichkeit zu tun hat, sondern im wesentlichen durch das Umfeld, in dem dieses Heft zu sehen ist, und den beschränkten Umfang der Publikation zustande gekommen ist. 
Zum Abschluß gilt es zu danken: Der Autorin und den Autoren für ihre Beiträge, der Redaktion und der Redaktionskommission der Geographica Helvetica für die Möglichkeit, dieses Heft zu gestalten, und nicht zuletzt Herrn H. LESER, der es möglich machte, daß diese Nummer der Geographica Helvetica allen Teilnehmerinnen und Teilnehmern am Deutschen Geographentag in Basel gratis abgegeben werden kann. Wir hoffen, da $\beta$ wir durch diese Publikationen einer größeren interessierten Leserschaft einen Einblick in einen interessanten und zukunftsträchtigen Teilbereich theoretischer und empirischer geographischer Forschungen, nämlich in den Grenzbereich zwischen Umwelt- und Regionalforschung, vermitteln können.

\section{Literatur}

BAHRENBERG, G. (1979): Von der Anthropogeographie zur Regionalforschung - eine Zwischenbilanz. In: Sedlacek P. (Hrsg.) (1979): Zur Situation der deutschen Geographie zehn Jahre nach Kiel. Osnabrücker Studien zur Geographie, Bd. 2, Osnabrück, 59-68.

BARTELS, D. (1968): Zur wissenschaftstheoretischen Grundlegung einer Geographie des Menschen. Beihefte zur Geographischen Zeitschrift, Nr. 18, Wiesbaden.

WACHTER, D. (1990): Externe Effekte, Umweltschutz und regionale Disparitäten - Begründung und Ausgestaltungsmöglichkeiten einer umweltbezogenen internalisierungsorientierten Regionalpolitik. Schriftenreihe Wirtschaftsgeographie und Raumplanung, Vol. 9, Geographisches Institut der Universität Zürich, Zürich. 